\title{
EXPLORANDO LA ENSEÑANZA \\ Y EL APRENDIZAJE EN LA \\ ESCUELA DE DERECHO DE \\ LA SALLE PACHUCA
}

Humberto Mejía Zarazúa 1*

\section{RESUMEN}

Se exponen algunas consideraciones teóricas, la metodología, los resultados, las conclusiones y sugerencias de un estudio exploratorio de las estrategias de enseñanza y de aprendizaje en la Escuela de Derecho de la Universidad La Salle Pachuca. El carácter exploratorio del estudio culminó en agosto de 2006 y las sugerencias, derivadas del proceso de investigación, pretenden ser elementos de análisis y reflexión para directivos, docentes y alumnos, con el fin de elevar la calidad educativa de dicha escuela.

\section{ABSTRACT}

Some theoretical considerations, the methodology, the results, the conclusions and suggestions of an exploratory study of the strategies of education and learning of the Right School La Salle Pachuca are exposed. The exploratory character of the study culminated in August of 2006 and the suggestions, derived from the investigation process, try to be elements of analysis and reflection by directors, educational and students, in order to elevate the educative quality of the mentioned school.

\section{INTRODUCCION}

La presente aportación condensa la información de un estudio exploratorio acerca de las estrategias de enseñanza y aprendizaje en la Escuela de Derecho de la Universidad La Salle Pachuca, tarea para la cual sumaron esfuerzos la dirección de la mencionada escuela y la Dirección de Posgrado e Investigación. El estudio se inscribe en la quinta línea de indagación del programa institucional de Investigación que se refiere a estudios sobre la perspectiva y prospectiva institucional. De acuerdo con el Plan de Desarrollo Institucional 2006-2010 de la Universidad La Salle Pachuca, la encuesta de percepción en la comunidad lasallista establece que el nivel académico se percibe como fortaleza y oportunidad, pero también como debilidad y amenaza, asimismo se considera como amenaza el que los egresados eventualmente no sean competentes en su ejercicio

profesional.

\footnotetext{
* Ingeniero Agrícola y Maestro en Ciencias de la Educación. Colaborador de la Dirección de Posgrado e Investigación de La Salle Pachuca, humberto_mz@hotmail.com
} 
Una de las finalidades de la Institución es que el nivel académico sea percibido por propios y extraños como una fortaleza y oportunidad institucional, toda vez que la Visión de La Salle Pachuca es: "Ser la mejor opción educativa en el Estado de Hidalgo para los niveles medio superior y superior porque en comunidad formamos personas comprometidas en la transformación de la sociedad". Por otro lado una de las líneas de acción es elevar el nivel académico y uno de sus objetivos específicos: "Realizar sistemáticamente actividades de autoestudio y evaluación". Este esfuerzo responde al mencionado objetivo (Universidad La Salle Pachuca, 2006 -Visión y Actividades Institucionales- s/p).

El objetivo general del estudio fue explorar las estrategias de enseñanza y de aprendizaje que se desarrollan en la Escuela de Derecho de la Universidad La Salle Pachuca a fin de sugerir elementos de discusión para el mejoramiento continuo del proceso educativo en la mencionada escuela.

\section{La educación superior en la actualidad}

En el ámbito educativo, el concepto de "educación para toda la vida" es ya un imperativo para las exigencias de un mundo globalizado donde cada vez es más patente la importancia de las competencias profesionales a fin de evitar la exclusión de los egresados de educación superior en el mercado laboral. Las universidades, por lo tanto, deben concienciar en sus alumnos que no basta con la conclusión exitosa de alguna licenciatura, sino que se tendrá que desarrollar fuera del ámbito escolarizado una educación permanente, flexible, diversa y accesible en el tiempo y en el espacio, no sólo como desarrollo personal, sino también por las inevitables mutaciones en el ejercicio profesional de cada disciplina, en el campo multidisciplinar y en la investigación. En este sentido la capacidad de "aprender a aprender" es fundamental en cualquier nivel educativo considerando que vivimos en un mundo que cambia rápidamente (Delors, 1996). Juan Carlos Tedesco (2000: 104), afirma que:

Ya no se trata simplemente de aprender determinado cuerpo de conocimientos e informaciones sino de aprender los mecanismos, las operaciones, los procedimientos que permitan actualizar nuestros conocimientos a lo largo de la vida. El desarrollo de la capacidad de aprender implica [...] disponer de amplias posibilidades de contactos con docentes que actúen como guías, como modelos, como puntos de referencia del proceso de aprendizaje.

Los recursos cognoscitivos cobrarán mayor relevancia a medida que pase el tiempo, por lo que la importancia de la educación superior también será cada vez mayor. Así, se requerirá mayor nivel en las competencias de los profesionales, capacidad de resolver nuevos problemas y de tomar iniciativas (Delors, 1996). El "aprender a aprender" se enmarca en el sentido asintótico con el que se produce conocimiento y la posibilidad de acceder a un enorme volumen de información. Actualmente la obsolescencia de competencias es un fenómeno permanente, lo cual obliga a procesos de reconversión profesional durante toda la vida activa laboral de los profesionales. Es fundamental desarrollar la capacidad de 
seleccionar, organizar y procesar la información que realmente se necesita, de tal manera que "... la educación ya no podrá estar dirigida a la transmisión de conocimientos y de informaciones sino a desarrollar la capacidad de producirlos y de utilizarlos" (Tedesco, 2000:

\section{La enseñanza del derecho}

Para Miguel Carbonell (2004), en general, en México existen bajos niveles en el proceso de enseñanza-aprendizaje en materia jurídica, aunque acepta que esta afirmación debe matizarse según el particular contexto al que se haga referencia, ya que acepta la existencia de "nichos" de excelencia y magníficos profesores de derecho. Sin embargo, reitera que generalmente los niveles educativos de las escuelas y facultades son preocupantes tanto en el Distrito Federal, como en el interior de la república. En el actual contexto, no sólo las clases magistrales deben ser la base para la formación de los juristas, sino cobran importancia los métodos expositivos y pedagógicos que fomenten la participación de los alumnos y les permitan desarrollar una visión crítica del sistema jurídico. Así, los estudios de caso, la lectura y discusión de textos, la presentación y discusión acerca de algún problema real, y el debate en aula argumentado con base en determinado punto de vista, pueden ser alternativas plausibles para crear estrategias complementarias a las exposiciones solitarias de los profesores. Lo anterior es fundamental para dotar a los alumnos de altos niveles argumentativos que les permitan defender determinadas posturas y no solamente basarse en el método memorístico.

Es de fundamental importancia para cualquier escuela o facultad brindar tanto información como formación, que además de desarrollar un sentido ético y de servicio a la sociedad, también atienda las necesidades del mercado y, por lo tanto, tome en cuenta las nuevas áreas temáticas que continuamente se desarrollan, sin descuidar el carácter general de una educación universitaria en el nivel de licenciatura. Por otro lado entre los problemas que Miguel Carbonell (2004) menciona respecto de las escuelas y facultades de derecho en torno a la enseñanza, se encuentran los siguientes:

a) Carencia de una visión completa del sistema jurídico al exagerar el énfasis endógeno del mundo jurídico, sin que los estudiantes reflexionen acerca de las dimensiones extrajurídi- cas (económicas, políticas y sociológicas, por ejemplo).

b) Insuficiente reconocimiento a la importancia de la creación y aplicación del derecho, de manera que muchos profesores sólo exponen lo que ya "estaba puesto".

c) No se desarrollan las capacidades y habilidades intelectuales necesarias para el ejercicio profesional, tales como el alto nivel argumentativo y otras. 
d) No se fomenta la posibilidad de aprender por sí mismos; es decir, es limitada la capacidad de investigación de los estudiantes, lo cual les acarrea problemas en su ejercicio profesio- nal para acceder a información relevante.

e) No se privilegia el aprendizaje significativo y no se fomenta la confianza de los alumnos en sus conocimientos y capacidades intelectuales.

Para Miguel Carbonell (2004) el tema de la enseñanza del derecho no ha sido suficientemente analizado en México, más aún cuando son tiempos de cambios políticos y jurídicos constantes. En este sentido, el mencionado autor afirma que:

... una de las modificaciones que, dentro del ámbito jurídico, todavía sigue pendiente es la del "modelo educativo» que se observa en las escuelas y facultades que enseñan derecho. La realidad amenaza con volver obsoletos un porcentaje importante de los conocimientos que se transmiten en esos centros educativos pero, al no darnos cuenta de ello, se mantiene el fuerte peso de la tradición, que no hace más que empujar de forma decidida hacia la obsolescencia. Urge revisar casi todo: desde los libros con los que se enseña, hasta los diseños curriculares, el perfil de los profesores y de investigación, las necesidades del mercado profesional, la vinculación de las universidades con la sociedad, la selección del alumnado, etcétera" (Carbonell, 2004, 72-73).

Miguel Carbonell (2004) también concluye que predomina una visión normativa del derecho, que se asocia a una idea de "pureza" metodológica, donde lo que acontezca fuera de las leyes y los códigos pertenecen a otras disciplinas y, por lo tanto, no son objeto de estudio. Asimismo es insuficiente el tiempo que se dedica a los tratados y jurisprudencia internacional; tampoco se toma en cuenta el análisis del régimen político y el financiamiento para la investigación es prácticamente nulo.

\section{Posicionamiento teórico}

Se entenderá por estrategias de enseñanza a los "procedimientos que el agente de enseñanza utiliza en forma reflexiva y flexible para el logro de aprendizajes significativos en los alumnos -es decir- son los medios o recursos para prestar ayuda pedagógica", y como estrategias de aprendizaje a los "procedimientos que el alumno utiliza en forma deliberada, flexible y adaptativa para mejorar sus procesos de aprendizaje significativo de la información" (DíazBarriga y Hernández, 2002: 141 y 430). Estas definiciones implican un posicionamiento a favor del aprendizaje significativo y del paradigma constructivista. Dicho aprendizaje según Coll, citado por Sanjurjo y Vera (2001: 29) se refiere a lo siguiente:

En síntesis, aprender significativamente supone la posibilidad de atribuir significado a lo que se aprende a partir de lo que ya se conoce. Este proceso 
desemboca en la realización de aprendizajes que pueden ser efectivamente integrados en la estructura cognitiva de la persona que aprende, con lo que se asegura su memorización comprensiva y su funcionalidad.

A su vez el constructivismo, se entiende como:

Confluencia de diversos enfoques psicológicos que enfatizan la existencia y prevalencia en los sujetos cognoscentes de procesos activos en la construcción del conocimiento, los cuales permiten explicar la génesis del comportamiento y el aprendizaje. Se afirma que el conocimiento no se recibe pasivamente ni es copia fiel del medio (Díaz-Barriga y Hernández, 2002:428).

Se considera importante que profesores y alumnos realicen de forma continua una revisión crítica de su propia práctica, tanto desde un análisis teórico pedagógico, como desde el contexto social e institucional en que se desarrolla el proceso de enseñanza y aprendizaje. Lo anterior supone algunas actividades que giran alrededor del acopio de información, discusión, reflexión, análisis y producción de propuestas por parte de alumnos, docentes y directivos.

Tanto el enfoque tradicional y el constructivista, tienen una relación directa con el tipo de aprendizaje. Por un lado tenemos el aprendizaje memorístico, y por otro el aprendizaje significativo; ambos se asemejan en tanto que ocurren en el contexto escolar pero se diferencian en que requieren distinto esfuerzo cognitivo y uso de los conocimientos previos. En general la distinción entre el enfoque tradicional y el constructivista, se basa en que el primero presenta información mediante el principio de que el conocimiento se transmite; en tanto el segundo, parte de que los alumnos pueden construir su conocimiento con base en el análisis, reformulación y evaluación de la información, así como a través de la interacción con sus compañeros y con los expertos (Gómez López, 2005).

La pedagogía tradicional se basa en la lección magistral, siendo el esquema básico la exposición-escucha-memorización-repetición. El método no se centra en el sujeto sino en el objeto de estudio que es el programa. Esto trae como consecuencia, entre otras cosas, que el conocimiento fomentado en la escuela contradice la manera en que se aprende fuera de ella, existiendo dicotomías como son el aprendizaje individual y el compartido, el conocimiento simbólico-mental y el físico-instrumental, ausencia de contexto y contextos concretos, por lo que la enseñanza tradicional a menudo es artificial, descontextualizada, poco significativa $\mathrm{y}$, por lo tanto, en contradicción con la vida real.

La educación tradicional privilegia el modelo conductista de manipulación del comportamiento mediante las recompensas y castigos, sin tomar en cuenta otro tipo de factores como las intenciones, pensamiento, esfuerzo o diferencias individuales de los alumnos. Lo anterior se refleja en la manera de "evaluar", premiar y castigar el desempeño de estudiantes, las ceremonias protocolarias de premiación, los cuadros de honor y hasta en los códigos de conducta y disciplina 
habituales. Bajo estas circunstancias "los alumnos responden por temor a la sanción, al castigo y a la reprobación, no porque exista un compromiso de conciencia o un deseo intrínseco por el trabajo escolar" (Díaz-Barriga y Hernández, 2002: 69).

Por otro lado, de acuerdo con Ausubel (citado por Díaz-Barriga y Hernández, 2002: 217) la enseñanza expositiva se recomienda si parte y se estructura con base en los conocimientos previos de alumnos de mayor edad (por ejemplo de nivel universitario) y se organiza de lo general a lo particular y de lo simple a lo complejo. Es decir, es posible obtener un aprendizaje significativo con base en la enseñanza expositiva; sin embargo, ésta por lo general se presenta básicamente de forma oral, sin retroalimentación de conocimientos y escasa interacción con los alumnos que suelen ser receptores pasivos de información, lo que provoca un aprendizaje superficial.

El constructivismo básicamente postula la importancia de los procesos activos de construcción del conocimiento más allá de lo que ofrece el entorno y, aunque existen diversos enfoques teóricos acerca del constructivismo, "comparten el principio de la importancia de la actividad mental constructiva del alumno en la realización de los aprendizajes escolares" (Díaz Barriga y Hernández, 2002: 29). Es decir, desde esta perspectiva se rechaza la concepción del alumno como ente pasivo, receptor y reproductor de "saberes" culturales. Pilar fundamental de este enfoque es el aprendizaje significativo. Los mencionados autores añaden que "la función del docente consiste en orientar y guiar la actividad mental constructiva de sus alumnos, a quienes proporcionará una ayuda pedagógica ajustada a su competencia". Esto supone que no existe una prescripción correcta acerca del "método" o única vía de enseñanza que debe seguir el profesor para el aprendizaje. Díaz-Barriga y Hernández (2002: 8-9) señalan un conjunto de actitudes y habilidades del docente constructivista:

- Convencerse de la importancia y del sentido del trabajo académico y de formación de conocimientos, habilidades, actitudes y comportamientos que está buscando promover en sus estudiantes.

- Respetar a sus alumnos, sus opiniones y propuestas, aunque no las comparta.

- Evitar imponer en un ejercicio de autoridad sus ideas, perspectivas y opciones profesionales y personales.

- Establecer una buena relación interpersonal con los alumnos, basada en los valores que intenta enseñar: el respeto, la tolerancia, la empatía, la convivencia solidaria, etcétera.

- Evitar apoderarse de la palabra y convertirse en un simple transmisor de información, es decir, no caer en la enseñanza verbalista o unidireccional. 
- Ser capaz de motivar a los alumnos y plantear los temas como asuntos importantes y de interés para ellos.

- Plantear desafíos o retos abordables a los alumnos, que cuestionen y modifiquen sus conocimientos, creencias, actitudes y comportamientos.

- Evitar que el grupo caiga en la autocomplacencia, la desesperanza o la impotencia, o bien, en la crítica estéril. Por el contrario, se trata de encontrar soluciones y construir alternativas más edificantes a las existentes en torno a los problemas planteados en y por el grupo.

La evaluación del proceso de aprendizaje es un tema complejo y siempre polémico, ya que involucra problemáticas internas y externas a la institución educativa. Sin embargo la anterior afirmación carece de sentido bajo una perspectiva tecnocrática que sólo plantee el construir instrumentos confiables de medición de determinados productos de aprendizaje, por ejemplo:

... Es común comprobar que los alumnos concurren a la escuela para obtener notas, en lugar de concurrir para aprender [...] El docente quedará atrapado en una gran maquinaria, como actor rutinario de atribuir calificaciones, e inconscientemente muchas veces utilizará la evaluación como refugio para salvar su prestigio, su lugar de poder, su autoridad. La relación docente-alumno queda así «prostituida» (Sanjurjo y Vera 2001: 129).

En las prácticas de evaluación predominantes subyace una concepción del alumno como un sujeto pasivo, receptivo y capaz de responder a estímulos. Respecto de la concepción del aprendizaje, ésta enfatiza la importancia de la memoria mecánica, por lo que la evaluación será recabar datos acumulados. Como el examen es el instrumento más importante de la evaluación, los alumnos van descubriendo cómo ocultar las cosas que ignoran. La evaluación mide productos no procesos y la función docente se reduce a ser el sujeto que corrige pruebas elaboradas por tecnócratas y planificadores. Los alumnos se preocupan más por obtener una buena calificación que por el proceso de autoconocimiento, observación y reflexión (Sanjurjo y Vera, 2001).

La contraparte de lo anterior sería una concepción de alumno como sujeto activo y el aprendizaje como un proceso permanentemente en construcción. De manera que la evaluación se considera como un ajuste pedagógico donde se tomarán en cuenta los procesos y los productos. Se evaluarán todos lo aspectos que intervienen en el proceso de enseñanza y de aprendizaje y participarán todos los sujetos implicados en tal proceso. Los instrumentos de evaluación deberán ser abiertos, globalizadores, flexibles, confiables y coherentes.

\section{Método y participantes}

La educación, más que una disciplina, es un campo de estudio que da cabida, para su investigación, a planteamientos metodológicos tanto cualitativos como 
cuantitativos. Este trabajo parte de que es posible superar la necesaria definición del método en tanto éste deba inscribirse en una u otra perspectiva. Se considera que es el objeto de estudio el que define la metodología específica que se habrá de utilizar. Así, para el presente trabajo se utilizó la observación no participante, la realización de dos entrevistas abiertas y una encuesta mediante la aplicación de un cuestionario.

En primer término se diseñó una guía de observación, la cual fue piloteada para su rediseño y aplicación, en ésta se establecieron categorías para las situaciones de clase, así como también se recurrió al registro anecdótico. Cabe aclarar que dado que se hizo una observación no participante sólo en determinadas asignaturas de determinados semestres, la interpretación de los resultados de ninguna manera se planteó como concluyente, en primera instancia por no ser una observación representativa respecto del total de asignaturas y semestres del programa académico; en segunda instancia, por el sesgo subjetivo que puede imprimir el observador a los resultados y a su interpretación. Sin embargo, esta fase, si bien, fue un primer acercamiento al objeto de estudio, dio pauta para determinar la orientación de las entrevistas abiertas.

Bassedas, Coll y otros (1984), citados por Leonor Buendía (1998: 158) mencionan que:

La observación perspicaz rigurosa y sistemática es el instrumento indispensable para comprender el comportamiento del alumno en el transcurso de las tareas de aprendizaje y para modificar su contenido y presentación en consecuencia. La observación adquiere casi papel preponderante y los problemas de qué observar y cómo observar se convierten en las cuestiones esenciales de la evaluación formativa...

Como se mencionó, el tipo de muestreo no fue estadístico, sino de manera intencional y focal, ya que se observó una asignatura de un grupo de primer semestre (Derecho Romano), una de tercer semestre (Teoría General de Estado), una de quinto semestre (Derecho Procesal Penal) y una de noveno semestre (Derecho Empresarial). De acuerdo con la clasificación de Evertson y Green (1997: 322-323), fue una observación descriptiva con categorías prefijadas, donde "El significado se considera específico del contexto [...] Se seleccionan conductas, acontecimientos y procesos que ocurren dentro de límites establecidos de forma natural", aunque con la limitación de no utilizar registros permanentes como la grabación o videograbación. La unidad de selección es el tiempo que dura la clase, y el registro se basa en una lista de confirmación en una unidad natural: el aula. Ésta si bien no es en sí misma natural, ha sido determinada por la realidad escolar.

Se realizaron también dos entrevistas abiertas, una al profesor de noveno semestre cuya clase fue observada y otra a una alumna de esa misma clase. Se consideró que, al no tener tiempo suficiente para realizar más entrevistas, hacerlo tanto a un profesor como a una alumna de, en ese momento el semestre más 
avanzado del segundo periodo de 2005, daría cuenta de puntos de vista con un panorama, por parte del profesor, con el conocimiento del alumnado que está cerca de concluir sus estudios; y por parte de la alumna, con la experiencia de recorrer casi todo el plan de estudios, así como el conocimiento de sus compañeros. La buena disposición del profesor y el prestigio de la alumna fueron decisivos para la elección. Si bien dos entrevistas son insuficientes para establecer generalizaciones, fueron la pauta para problematizar el objeto de estudio, es decir, con base en ellas y en el proceso de observación no participante se definieron aspectos de indagación basados en hipótesis, para que mediante éstas y el marco teórico, se diseñara un cuestionario y se aplicara la encuesta. Los aspectos de indagación fueron: aprendizaje significativo; modelo conductista; capacidad de interpretación y análisis de documentos y ordenamientos jurídicos; evaluación; negociación de la calificación; análisis contextual de la normatividad; desarrollo de habilidades para la investigación; dictado, debate, crítica y reflexión; aprovechamiento pedagógico de grupos reducidos; y escuela y desarrollo profesional.

La encuesta se aplicó al 87 por ciento del alumnado durante el periodo del primero al seis de junio de 2006, para lo cual se elaboró una base de datos mediante el programa de análisis estadístico SPSS, se capturó y procesó la información obtenida para realizar la interpretación a la luz del marco teórico. El piloteo del cuestionario y la aplicación de la encuesta estuvo a cargo de la Escuela de Derecho. El cuestionario se conformó con una parte de preguntas cerradas, otra parte con afirmaciones que debían calificarse como verdaderas o falsas, otra más con afirmaciones para la escala de medición tipo Likert y finalizó con la pregunta abierta: Por último por favor escribe alguna(s) recomendación(es) que consideres necesaria(s) para elevar el nivel académico en la Escuela de Derecho de la Universidad La Salle de Pachuca. Es necesario tomar en cuenta que el estudiantado, al estar inmerso en lógicas de cotidianidad y perspectivas personales, pueden ofrecer respuestas a un cuestionario, sin que éstas necesariamente correspondan a las intenciones que el diseñador planificó (Coulon, 1995), por lo que la interpretación de los resultados tiene que involucrar el aspecto contextual, en este caso conocido por los docentes y directivos de la Escuela de Derecho.

La población encuestada fue de 80 alumnos de los cuales uno de octavo semestre no consignó datos de sexo, ni edad, por lo que de 79 estudiantes tenemos que 42 son mujeres $(53.2 \%)$ y 37 hombres $(46.8 \%)$ con un promedio de edad de casi 21 años.

\section{Resultados}

La mayoría de los estudiantes de derecho tienen la meta de aprender y consideran que sus conocimientos los obtienen en relación con los conocimientos previos. Las respuestas abiertas destacan el papel del catedrático, el esfuerzo personal y la vida cotidiana como fuentes de conocimiento. Opinan (81 por ciento) que para aprobar sus exámenes es necesario analizar, interpretar, integrar y 
sintetizar información, y sólo el 16 por ciento piensa que se requiere de buena memoria. Lo anterior indicaría que un aspecto importante de la evaluación (el examen) supone la exposición de conocimientos de manera amplia y abierta, más que en la exposición memorística de datos.

La mayoría del alumnado no tiene reparo en preguntar al profesor sus dudas respecto de su explicación, lo que indicaría la disposición del docente para hacer las aclaraciones pertinentes acerca de su clase. Cabe aclarar que este tipo de alumnos (77 por ciento) tiene un aprovechamiento escolar traducido en promedio de calificaciones de 8.48, en tanto los 11 alumnos (14 por ciento) que prefieren preguntar a sus compañeros o indagar por cuenta propia tienen un promedio de 7.93 de calificación y los que esperan a que otros pregunten por ellos tienen un promedio de 7.95. Sin ser conclusivos podría pensarse que hay una correlación entre "bajas" calificaciones y personalidad retraída y/o introvertida del estudiante, o bien "miedo al ridículo" asociado con una cultura estudiantil que involucra la "burla" hacia preguntas aparentemente "obvias". Este aspecto habría que profundizarlo si se quisiera establecer un sistema de "tutorías" en la Escuela de Derecho. Por otro lado más de la mitad del alumnado (68 por ciento) considera que sus profesores no tienen problema en el manejo de la información relativa a la asignatura que imparten.

En el reporte de las sesiones de observación se escribió que hubo actitudes de algunos alumnos que hacían pensar que para preparar sus exámenes requerían de "certidumbre", no sólo respecto del contenido temático a cubrir, sino de "tener" ese contenido a la mano ya sea en los apuntes escolares (primer semestre) o en determinado libro (noveno semestre). Así, el resultado del cuestionario sobre este aspecto podría ser revelador de una limitada capacidad de investigación del estudiantado, ya que el 52 por ciento siente mayor seguridad para preparar un examen si tiene anotado en sus apuntes el contenido temático correspondiente, es decir, probablemente no se fomente la posibilidad de aprender por sí mismos y acceder a información relevante. Este supuesto tendría que estar relacionado con el tipo de exámenes que se aplican. Por lo anterior, se considera relevante revisar si es necesario trabajar en las habilidades y competencias para la investigación jurídica, ya que esto es fundamental para el buen desarrollo del futuro ejercicio profesional. Cabe aclarar que el alumnado que expresa mayor seguridad para preparar un examen a partir del conocimiento de la temática y su estudio en diversas fuentes de información (48 por ciento) tiene un promedio ligeramente mayor que el resto de sus compañeros ( 8.50 y 8.28 respectivamente).

En el registro anecdótico de la fase de observación, se anotó que al parecer no se desarrollaba la habilidad expositiva del alumnado, ya que la lectura (cuando exponía el alumno) era el recurso más empleado. Sin embargo, la percepción del alumnado es diferente, pues el 67 por ciento afirma utilizar diapositivas y acetatos que contienen un guión de exposición con base en el cual el estudiante explica determinado tema. Por lo anterior, para la interpretación a este respecto es fundamental el criterio de los profesores acerca de la veracidad de la autopercepción del estudiantado y con base en ello, tomar las medidas 
conducentes para desarrollar la habilidad argumentativa y expositiva que se considera crucial en el ejercicio de la abogacía.

No sólo la clase magistral debe ser la base para la formación de abogados, sino que es importante desarrollar en el estudiantado una visión crítica del sistema jurídico. Por ello, si bien es cierto que la mayoría del alumnado (60 por ciento) considera que se fomenta su sentido crítico, es de considerar el 37 por ciento que opina lo contrario, más aún tomando en cuenta que son estudiantes que no contestan por reacción a una deficiente trayectoria académica, de hecho su promedio escolar es ligeramente mayor que los primeros (8.46 el 37 por ciento y 8.31 el 60 por ciento). Lo anterior sugiere que sería recomendable enfatizar la ampliación de criterios jurídicos vistos en las clases; sin embargo, como en otras afirmaciones, se considera que el cuerpo docente y directivo de la Escuela de Derecho debe reflexionar y concluir al respecto.

La percepción del alumnado respecto al porcentaje de profesores que son especializados en la asignatura que imparten es la siguiente: el 37 por ciento del estudiantado (el más alto de los resultados) opina que el porcentaje referido oscila entre el 51 al 75 por ciento; seguido por el 28 por ciento que opina que entre el 76 al 100 por ciento de los docentes tienen las mencionadas capacidades; es decir más de la mitad de estudiantes (65 por ciento), consideran que más de la mitad de profesores de derecho tienen experiencia docente y son especializados en la asignatura que imparten.

Aun cuando la indagación sobre el autoconcepto del alumnado, ya sea como sujeto pasivo, receptivo y capaz de responder a estímulos; o como sujeto activo que ve el aprendizaje como un proceso permanente en construcción, se puede pensar que es predecible, se consideró importante explorar este rubro. La segunda concepción la tiene el 67 por ciento del alumnado, mientras que el 20 por ciento tendría la primera. El 13 por ciento restante optó por expresar una respuesta abierta en la que básicamente plantean que se preocupan tanto por el proceso de conocimiento, análisis y reflexión, así como por la calificación (estos 12 alumnos en conjunto tienen un mayor promedio que el resto: 8.73).

Para tener una visión integral del sistema jurídico es necesario reflexionar acerca del contexto extrajurídico. En este sentido la mayoría del alumnado (84 por ciento) considera estar al tanto de la situación política, económica y social de México, a través de revistas, periódicos, noticiarios y contenido de algunas clases en la escuela.

Se ha mencionado la importancia para la formación de los abogados de los métodos expositivos y pedagógicos que fomenten la participación del alumnado y le dote de altos niveles argumentativos. Relativo a lo anterior el estudiantado opina que la exposición de los profesores ocupa dos tercios del tiempo de clase y el resto el alumno. Es importante la interpretación del colectivo de la Escuela de Derecho a este respecto; sin embargo, se considera que se podría reflexionar 
acerca de la conveniencia de incrementar la participación de los estudiantes en el desarrollo de las clases.

El aprendizaje memorístico y el estudio y análisis de casos son las estrategias de aprendizaje más comunes en opinión del alumnado y, con menor frecuencia, se utiliza la lectura y discusión de textos, los debates en aula y la investigación de temas. Esto podía sugerir que es necesario fomentar aún más las estrategias alternativas a las exposiciones de los profesores

Ante la pregunta: ¿qué tipo de exámenes, en general, te aplican según tu punto de vista?, la respuesta del alumnado de derecho asigna el mayor porcentaje a las llamadas pruebas objetivas, las cuales reflejan una concepción mecanicista del aprendizaje, le sigue en importancia las pruebas tradicionales y las pruebas semiestructuradas. Las pruebas a libro abierto y el trabajo grupal para la evaluación obtienen un porcentaje poco significativo. Este resultado invita a reflexionar acerca del proceso de evaluación que se practica en la Escuela de Derecho respecto de la pertinencia de promover más la reflexión, valoración y análisis de la información, que la exposición de conocimientos memorísticos.

En los resultados de las afirmaciones que habrían de calificarse como falsas o verdaderas se aprecia que la mayoría del alumnado considera que las actitudes y habilidades del cuerpo docente corresponden a una posición constructivista (67 por ciento del alumnado), excepto en lo que se refiere a cierto autoritarismo derivado de la imposición de ideas, perspectivas y opiniones profesionales y personales, por lo que se considera que habría que reflexionar acerca de este aspecto.

La parte del cuestionario diseñada mediante la técnica de medición de actitudes a través de la escala de Likert, es la que tuvo resultados más cuestionables debido quizá a que las afirmaciones no estuvieron lo suficientemente bien diseñadas. El resultado sugiere que existe una indefinición por parte del alumnado acerca de los siguientes aspectos de indagación: existencia de aprendizaje significativo; modelo conductista de manipulación del alumnado; forma de evaluación; desarrollo de habilidades para la investigación; dictado versus crítica, debate y reflexión; aprovechamiento pedagógico de tener grupos relativamente reducidos. Por otro lado, sí hubo un posicionamiento respecto a que se considera que dentro la Escuela de Derecho se desarrolla la capacidad de interpretación y análisis de documentos y ordenamientos jurídicos; así como la existencia de interés por el análisis del contexto político, social y económico de la normatividad vigente. También se tiene conciencia de que, si bien, la escuela es un gran apoyo para adquirir las competencias necesarias para un adecuado desarrollo profesional, es insuficiente $y$, además del aspecto práctico, es necesario un esfuerzo adicional respecto de lecturas, análisis y reflexión. Por otro lado, se interpreta que no existe una cultura de "negociación de la calificación". 
Parte relevante y fundamental del cuestionario fueron las respuestas a la pregunta abierta. La categoría que más sugerencias tuvo fue la de docentes, en la que destaca la recomendación de contratar profesores expertos en la asignatura que imparten, e incrementar sus competencias como docentes, así como disminuir el autoritarismo. La segunda categoría se refiere a aspectos relacionados con la enseñanza y aprendizaje en la que destaca la recomendación de asistir a eventos tales como seminarios, congresos y ponencias, así como el incremento del aprendizaje práctico. La tercera categoría se refiere a aspectos del alumnado y básicamente se sugiere que los estudiantes sean tomados en cuenta para la toma de decisiones. Una crítica relevante se centra en que el diseño de horarios no correspondía a las necesidades del estudiantado, en particular lo que concierne a inglés. Respecto de la categoría de la evaluación, se enfatiza el diseño de un sistema de exención. La categoría de normatividad se considera que no tuvo relevancia.

Si consideramos las principales demandas del alumnado, independientemente de la categoría a la que se le haya adscrito, se obtiene que las principales demandas son la contratación de profesores expertos en la materia, la asistencia a eventos, el desarrollo de competencias docentes (respecto a las estrategias de enseñanza) y el tomar en cuenta la opinión del alumnado para la toma de decisiones. Estas demandas cubren el 42 por ciento del total de las registradas.

\section{CONCLUSIONES}

Las presentes conclusiones expresan un punto de vista que busca poner a discusión de directivos, profesores y alumnos de la Escuela de Derecho de la Universidad La Salle Pachuca algunas consideraciones del proceso de enseñanza y aprendizaje. Es importante reiterar que, lo expresado en esta investigación no responde a una interpretación por parte del personal de la mencionada escuela sino del autor del presente artículo. Asimismo, los resultados, tanto en las diferentes fases del estudio como en las diferentes secciones del cuestionario, no apuntan a conclusiones unívocas (hay ciertos resultados contradictorios), sino a puntos de discusión, de ahí su carácter exploratorio. Se considera que lo anterior se debe tanto a debilidades metodológicas del presente trabajo, como a los aspectos subjetivos inherentes en la percepción del alumnado.

En general, las actitudes y habilidades de los docentes corresponden a una posición constructivista de acuerdo con la parte de preguntas cerradas del cuestionario, aunque en la parte de la escala Likert la opinión es de indefinición a este respecto. Los nuevos conocimientos a adquirir se obtienen a partir de los conocimientos previos y la mayoría del alumnado pregunta a sus profesores si tienen algunas dudas acerca del contenido de la clase; éstos, en general tienen experiencia docente y buen dominio de las asignaturas que imparten, aunque ciertas limitaciones para guiar el proceso de autoaprendizaje del alumnado, de ahí que sientan mayor seguridad para preparar un examen si tienen en sus apuntes el contenido temático de las asignaturas. En las respuestas a la pregunta abierta se 
expresa la inquietud de que se contraten sólo profesores expertos en la asignatura que imparten.

Los docentes respetan las opiniones y propuestas del estudiantado, aunque no las comparten; pero, sus ideas, perspectivas y opiniones profesionales y personales imponen un ejercicio de autoridad. De cualquier modo la relación que establecen con el alumnado se basa en el respeto y la tolerancia. Asimismo, son capaces de motivar e interesar a los estudiantes respecto de la temática de clase. Se recomienda revisar la pertinencia de ejercer cierto "autoritarismo" por parte de algunos catedráticos, ya que, paradójicamente con lo antes mencionado, es un aspecto expresado por el alumnado en las respuestas a la pregunta abierta.

La participación y exposición del estudiantado en clase, de acuerdo con su percepción, cubre la tercera parte de las sesiones. Por otro lado la estrategia más utilizada es la enseñanza que se basa en el aprendizaje memorístico (específico resultado que contradice la posición constructivista del docente), dejando en segundo plano otras estrategias como el estudio y análisis de casos, la investigación y el debate. De acuerdo con el resultado del cuestionario, por un lado el alumnado opina que la evaluación mediante exámenes se basa en el análisis, interpretación, integración y síntesis de la información y, por otro, la opinión mayoritaria es que en los exámenes se solicitan respuestas únicas, cuya selección es a veces al azar. Esta ambivalencia podría suponer una revisión en el método de evaluación de los profesores.

Los estudiantes consideran que en La Salle Pachuca sí existe la capacidad de interpretación y análisis de documentos y ordenamientos jurídicos, además de que tienen conciencia de la necesidad de realizar un esfuerzo adicional de lectura, análisis y reflexión más allá de lo que hacen en la escuela para tener éxito profesional. Aun cuando la autopercepción de los estudiantes está ligada a considerarse preocupados más por el conocimiento, el análisis y la reflexión que por obtener una buena calificación, y opinan tener conocimiento de la situación política, económica y social de México; se considera importante asegurar la capacidad expositiva y argumentativa del alumnado, así como la discusión de varias posturas (o autores) a fin de ampliar su criterio jurídico. Por otro lado el estudiantado considera que es necesario asistir a conferencias, seminarios, ponencias y congresos, es decir, a eventos fuera de La Salle Pachuca. Este aspecto se considera de suma importancia, ya que podría revelar una tendencia académica endógena que limitaría el desarrollo del criterio jurídico del estudiantado, al no abrirse a otras opiniones además de las del cuerpo docente. Asimismo, los estudiantes piensan que su opinión no es lo suficientemente tomada en cuenta para la toma de decisiones.

Finalmente se pone a consideración de la Escuela de Derecho las siguientes sugerencias: 
- Incrementar las habilidades para la investigación del alumnado.

- Incrementar las habilidades expositivas y argumentativas del alumnado.

- $\quad$ Promover el debate en el aula.

- Incorporar en la evaluación procesos de mayor reflexión, análisis e interpretación del conocimiento.

- Reflexionar acerca de la percepción del alumnado respecto al "autoritarismo" de algunos profesores.

- Fomentar la asistencia del alumnado a eventos académicos fuera de La Salle.

- Reflexionar acerca del diseño de procesos para conocer y tomar en cuenta la opinión del alumnado para la toma de decisiones.

- Contratar profesores con experiencia laboral en aspectos relacionados con la asignatura que impartirán.

- Capacitar a los docentes para ampliar su panorama respecto de estrategias de enseñanza y aprendizaje (incremento de habilidades docentes)

\section{REFERENCIAS}

BUENDÍA, L., Pilar Colas y Fuensanta Hernández (1998), Métodos de Investigación en psicopedagogía, Madrid: Mc Graw Hill UNAM.

CARBONELL, Miguel (2004), La enseñanza del derecho, México: Porrua,

COULON, Alain (1995), Etnometodología y educación, Barcelona: Paidós Educador

DELORS, Jacques (1996), La educación encierra un tesoro, México: Ediciones Unesco.

DÍAZ-BARRIGA, F. y Gerardo, H. (2002), Estrategias docentes para un aprendizaje significativo, México: Mc Graw Hill

EVERSTON, C. y Judith Green (1997), La observación como indagación y método, en: Wittrock, Merlin (Coord.), La investigación de la enseñanza, II. Métodos cualitativos y de observación, Barcelona: Paidos.

GÓMEZ, Felipe (2005), Comparación de una propuesta pedagógica universitaria con las prácticas cotidianas en las aulas, en: Revista Mexicana de 
Investigación Educativa 165, http://www.cesu.unam.mx/rmie/num24/24investig5.pdf. Consultado el 23 de agosto de 2005.

SANJURJO, Liliana y Vera, Ma Teresita (2001), Aprendizaje significativo y enseñanza en los niveles medio y superior, Rosario, Argentina: Homo Sapiens Ediciones.

TEDESCO, Juan Carlos (2000), Educar en la sociedad del conocimiento, México: FCE.

Universidad La Salle Pachuca (2006), Plan de Desarrollo Institucional 20062010, Pachuca, Hidalgo, México 